\title{
A Novel Molecularly Imprinted Polymer for the Selective Removal of Chlorophyll from Heavily Pigmented Green Plant Extracts prior to Instrumental Analysis
}

\author{
Bareki Shima Batlokwa, ${ }^{1}$ Janes Mokgadi, ${ }^{2}$ Ronald Majors, ${ }^{3}$ \\ Charlotta Turner, ${ }^{4}$ and Nelson Torto ${ }^{2}$ \\ ${ }^{1}$ Department of Earth \& Environmental Science (Chemistry Unit), Botswana International University of Science \& Technology, \\ P.O. Box 041, Bontleng, Gaborone, Botswana \\ ${ }^{2}$ Department of Chemistry, Rhodes University, P.O. Box 94, Grahamstown 6140, South Africa \\ ${ }^{3}$ Agilent Technologies, Inc., 2850 Centerville Road, Wilmington, DE 19808, USA \\ ${ }^{4}$ Department of Chemistry, Center for Analysis and Synthesis, Lund University, P.O. Box 124, 22100 Lund, Sweden
}

Correspondence should be addressed to Bareki Shima Batlokwa; batlokwab@biust.ac.bw

Received 11 January 2013; Accepted 16 March 2013

Academic Editor: Chiyang $\mathrm{He}$

Copyright (C) 2013 Bareki Shima Batlokwa et al. This is an open access article distributed under the Creative Commons Attribution License, which permits unrestricted use, distribution, and reproduction in any medium, provided the original work is properly cited.

\begin{abstract}
A novel molecularly imprinted polymer (MIP) powder designed for the selective removal of interfering chlorophyll pigment from heavily pigmented green plant extracts during pesticide residue analysis or analysis for bioactives in natural product research is reported. The polymer powder imprinted with chlorophyll $a$ was synthesized by copolymerizing the functional and crosslinking monomers, methacrylic acid (MAA) and ethylene glycol dimethacrylate (EGDMA), respectively. During batch rebinding experiments, the MIP effectively removed chlorophyll from neat chlorophyll standards, green medicinal plants, and spinach extracts with determined absorbance as high as 2.501 absorbance units ( $\mathrm{Au}$ ) to as low as $0.084 \mathrm{Au}$ after the optimum quantity of the MIP $\left(170 \mathrm{mg} \mathrm{mL}^{-1}\right)$ was added as the adsorbent prior to instrumental analysis. The determined $0.084 \mathrm{Au}$ absorbance was far below the absorbance of $2 \%$ chlorophyll $(0.401 \mathrm{Au})$ regarded as the cut-off point for interfering chlorophyll. Thus the newly developed polymer presents itself as a suitable adsorbent for the selective removal of chlorophyll from heavily pigmented, chlorophyll containing extracts.
\end{abstract}

\section{Introduction}

Chlorophyll the green pigment found in all photosynthetic plants as well as in some bacteria, often interferes with the quantitative analysis of bioactives in natural product research or in pesticide residue analysis [1]. For example, in the Quick Easy Cheap Effective Rugged Safe (QuEChERS) technique, extracts of green vegetables like spinach which are highly pigmented with chlorophyll, challenge the analytical portion of the method during pesticide residue analysis [2]. Such a type of matrix introduces severe interferences in the analytical separation step. Moreover, very "dirty" samples exhibit a noxious property of strongly influencing the background ion current in mass spectrometry (MS) detectors, thus reducing their sensitivities [3]. Of consequence, quantitative analysis can be achieved only after extensive clean-up step(s) [4]. Currently graphitized carbon black (GCB) is employed in the dispersive SPE kits of QuEChERS in order to remove the high levels of the green pigment from the vegetable matrix. However GCB does not only remove chlorophyll, but it also retains pesticides with planar structures resulting in poor recovery and precision [5]. Moreover it is very expensive.

In order to address the challenges of GCB on planar pesticides or bioactives recoveries, a sample preparation step employing cheap, selective materials should be developed so that only the interfering chlorophyll is removed prior to pesticide residue or bioactive analysis. An example of such materials is the molecular imprinted polymers (MIPs). They 
are synthetic polymers with selective recognition sites that have been created during imprinting with a template which is usually the target molecule. MIPs have seen increased use in contaminant or trace analysis as suitable materials for applications where analyte selectivity is essential [6]. They have been shown to be suitable as sorbent materials for solid phase extraction [7-12], hence providing a selective clean-up and preconcentration step for samples normally associated with complex matrices.

This paper presents preliminary results for the preparation and evaluation of the effectiveness of a novel, simply and cheaply prepared MIP for the specific removal of chlorophyll from green plants extracts relative to the performance offered by the expensive, nonspecific GCB which has been in use.

\section{Experimental}

2.1. Chemicals. Chlorophyll a standard, hexachlorobenzene (HCB), dichlorodiphenyldichloroethylene (DDE), dichlorodiphenyldichloroethane (DDD), graphitized carbon black (GCB), methacrylic acid (MAA), ethylene glycol dimethacrylate (EGDMA), dichloromethane (DCM), HPLC grade methanol and azobisisobutyronitrile (AIBN), and sodium acetate/acetic acid buffer were supplied by Sigma-Aldrich (Saint Louis, MO, USA). Fresh spinach was bought from a local shop (Grahamstown, South Africa). Reagents used were of analytical grade or better. All water used was purified using a Direct Q 3UV Millipore system. Tswii (Nymphaea lotus) and Makgonatsotlhe (Acalypha villicaulis) leaves are traditional medicines collected from the Okavango Delta area in Botswana (Okavango Delta, Botswana).

2.2. Instrumentation and Apparatus. A lambda 25 PerkinElmer spectrophotometer, by Perkin-Elmer, (Santa Clara, CA, USA) was used to detect the concentration of chlorophyll (at $680 \mathrm{~nm}$, using a $1 \mathrm{~cm}$ cell) in every washing during template removal and the supernatant after addition of the MIP during batch rebinding experiments. Agilent GC-6820 (Santa Clara, CA, USA) equipped with ${ }^{63} \mathrm{Ni}$ electron capture detector and DB-5 MS fused-silica capillary column $(30 \mathrm{~m}$ length, $0.25 \mathrm{~mm}$ internal diameter, and $0.25 \mu \mathrm{m}$ film thickness) were employed in the separation and determination of the concentrations of planar pesticides that were coextracted with the interfering chlorophyll. GC analytical conditions used were as follows: oven temperature programming: $50^{\circ} \mathrm{C}$ (hold time: $1 \mathrm{~min}$ ), raised to $145^{\circ} \mathrm{C}$ at $30^{\circ} \mathrm{C} \mathrm{min}^{-1}(3 \mathrm{~min}$ ), to $260^{\circ} \mathrm{C}$ at $20^{\circ} \mathrm{C} \mathrm{min}^{-1}(3 \mathrm{~min})$, and finally to $300^{\circ} \mathrm{C}$ at

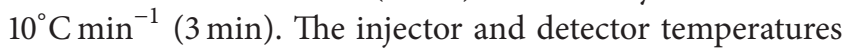
were held at $280^{\circ} \mathrm{C}$ and $300^{\circ} \mathrm{C}$, respectively. Helium flowing at $2 \mathrm{~mL} \mathrm{~min}^{-1}$ was used as the carrier gas while nitrogen was the make-up gas at $1.3 \mathrm{~mL} \mathrm{~min}^{-1} .1 \mu \mathrm{L}$ sample was injected in the splitless mode. An MSE Mistral 1000 by Sanyo Gallenkamp, (Loughborough, England) was employed for centrifugation. Standard Test sieves by Retsch GmbH \& Co. (Haan, Germany) were used to obtain the average size of the polymer particles by screen analysis.

2.3. Preparation of the Chlorophyll Molecular Imprinted Polymer (MIP). For the preparation of the molecularly imprinted polymers (MIPs), chlorophyll $a$, MAA, EGDMA, and AIBN in the mole ratio $1: 5: 25: 0.4$ were dissolved in $35 \mathrm{~mL}$ of DCM, respectively. The mixture was purged with nitrogen for $10 \mathrm{~min}$ and refluxed on an oil bath at $75^{\circ} \mathrm{C}$ for $9 \mathrm{~h}$. The resultant rigid polymer was ground to a powder of particles sizes of less than $45 \mu \mathrm{m}$ in diameter after which the template was thoroughly removed following the pressurized hot water extraction approach proposed by Batlokwa et al. [13] with slight modifications. Thereafter, the particles were left to dry in open air overnight. A reference polymer referred to as a nonimprinted polymer (NIP), without the imprinting template chlorophyll $a$, was prepared with a similar procedure to provide for the control in the rebinding experiments.

2.4. Optimization of MIP Quantity Needed for Maximum Removal of Chlorophyll. Increasing quantities of MIP were added to $5 \mathrm{~mL}$ aliquots of $10 \%(\mathrm{w} / \mathrm{v})$ chlorophyll. They were then equilibrated for $24 \mathrm{~h}$ at $\mathrm{pH} 7$ in sodium acetate/acetic acid buffer and centrifuged for $5 \mathrm{~min}$. The absorbance of chlorophyll in the supernatant was determined with a UV spectrophotometer until there were constant readings with further additions of the MIP. Leaching experiments from the MIP particles were performed following a procedure by Batlokwa et al. [13] to confirm the absorbances determined in the supernatants. The experiments were carried out in triplicates, and the average $\%$ bound to the MIP was calculated. Statistical methods were used to determine the mean values and the percentage standard deviations (\% RSD). From the values, a plot of absorbance against quantity of MIP added as the adsorbent was constructed.

2.5. Optimization of Time Needed for Maximum Removal of Chlorophyll. Following the procedure for optimization of quantity, absorbance of chlorophyll in the supernatant was monitored at $5 \mathrm{~min}$ intervals until a constant value of absorbance was reached. This marked the optimum time needed for the polymer to bind maximally.

2.5.1. Selectivity Experiments. Employing the optimized conditions, competitive and nonselectivity experiments were performed. Both the imprinted and nonimprinted polymers were used to obtain two sets of experimental data. $850 \mathrm{mg}$ polymer material or GCB was added to $5 \mathrm{~mL}$ aliquots containing a $10 \%(\mathrm{w} / \mathrm{v})$ chlorophyll standard, $1 \mu \mathrm{g} \mathrm{mL}^{-1}$ of each of the pesticides, HCB, DDD, and DDE. The same procedure was repeated for a mixture of chlorophyll and all the pesticides in order to cater for their coexistence. Column GC-ECD was employed in the seperation and determination of the concentrations of the pesticides.

2.6. Effectiveness of the MIP, NIP, and GCB in Removing Chlorophyll from Spinach and Traditional Medicine Plant Leaves Extracts. To obtain chlorophyll from spinach and traditional medicine plant leaves, $10 \mathrm{~g}$ of each was blended with $80 \mathrm{~mL}$ methanol for $5 \mathrm{~min}$, and the green supernatants were used as test samples. The initial absorbance for each of the extracts was determined after which the optimized quantity of MIP, $850 \mathrm{mg}$, was added to $5 \mathrm{~mL}$ extracts, equilibrated for $5 \mathrm{~min}$, 
TABLE 1: A Comparison of selectivity of various adsorbents in specifically binding chlorophyll and/or pesticides at optimized conditions.

\begin{tabular}{|c|c|c|c|}
\hline Analyte/interferent & $\begin{array}{c}\text { \% bound to MIP } \\
n=3\end{array}$ & $\begin{array}{c}\text { \% bound to GCB } \\
n=3\end{array}$ & $\begin{array}{c}\text { \% bound to NIP } \\
n=3\end{array}$ \\
\hline Chlorophyll (spinach extract) & $99.86(0.72)$ & $99.92(1.01)$ & $2.96(0.19)$ \\
\hline Chlorophyll (Nymphaea lotus extract) & $99.90(0.64)$ & $99.95(0.83)$ & $3.66(0.09)$ \\
\hline Chlorophyll (Acalypha villicaulis extract) & $99.86(0.97)$ & $99.88(1.06)$ & $2.42(0.04)$ \\
\hline $\mathrm{HCB}$ & $\mathrm{nq}$ & $90.41(1.67)$ & $3.61(0.28)$ \\
\hline DDE & $\mathrm{nq}$ & $89.97(0.93)$ & $3.44(0.01)$ \\
\hline DDD & $\mathrm{nq}$ & $91.28(1.38)$ & $3.29(0.14)$ \\
\hline \multicolumn{4}{|l|}{ Mixture } \\
\hline Chlorophyll & $99.79(1.03)$ & $88.94(0.73)$ & $3.22(0.36)$ \\
\hline $\mathrm{HCB}$ & $\mathrm{nq}$ & $94.52(1.84)$ & $3.49(0.92)$ \\
\hline DDE & $\mathrm{nq}$ & $90.26(1.51)$ & $2.79(0.02)$ \\
\hline DDD & $\mathrm{nq}$ & $93.08(1.96)$ & $3.58(0.16)$ \\
\hline
\end{tabular}

Values in parenthesis are \% RSD, and nq is for not quantifiable.

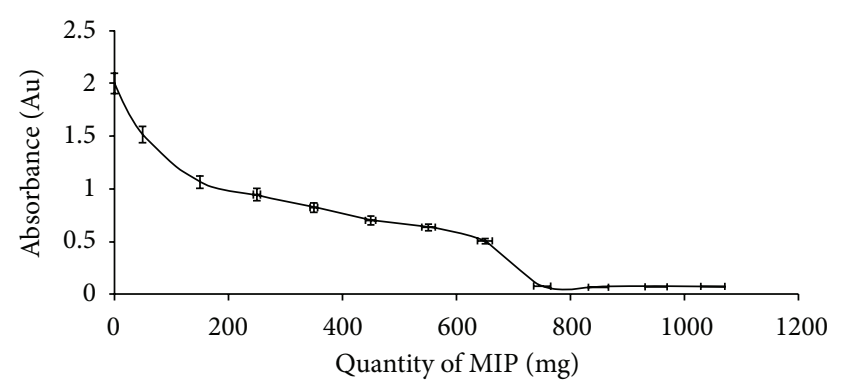

FIGURE 1: Optimization of the quantity of MIP needed to remove maximum chlorophyll in $24 \mathrm{~h}$ at $\mathrm{pH}$ 7. $n=3$.

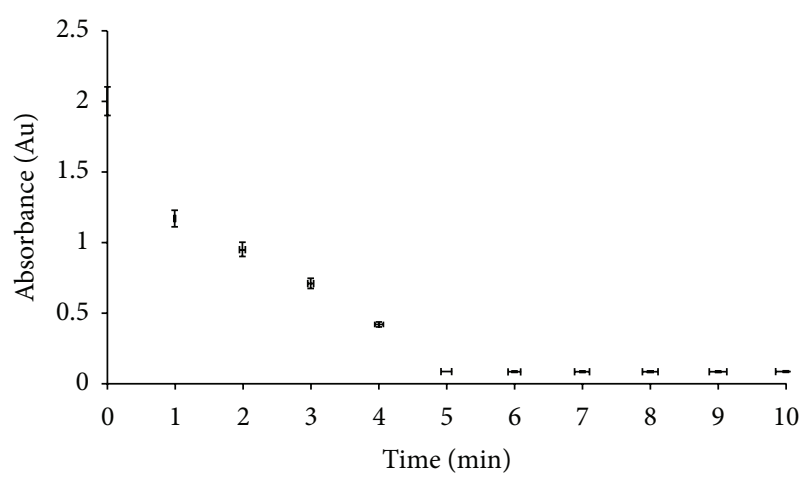

Figure 2: Optimization of the time needed for maximum removal of chlorophyll, at optimal MIP quantity of $850 \mathrm{mg}$ and $\mathrm{pH}$ 7. $n=3$.

and then centrifuged for another $5 \mathrm{~min}$. The absorbance of the supernatants was then determined and compared to that of a $2 \%$ chlorophyll standard, below which chlorophyll is claimed not to interfere with analysis [1].

\section{Results and Discussion}

3.1. Optimized Quantity of MIP Needed for Maximum Removal of Chlorophyll. The absorbance (concentration) of chlorophyll decreased with an increase in the quantity of MIP added until an optimal value of $850 \mathrm{mg}$ in the $5 \mathrm{~mL}$ chlorophyll standard solutions (see Figure 1) was reached. According to Mastovska and Lehotay and our own findings, $300 \mathrm{mg}$ of GCB (550 mg less than the quantity of the MIP we prepared) was needed to remove an equivalent concentration of chlorophyll [14]. The prepared polymer removed chlorophyll up to a very low absorbance $(0.081 \mathrm{Au})$ when compared to the absorbance of $0.399 \mathrm{Au}$ that is said to interfere with the analysis [1], which is the absorbance of a $2 \%(\mathrm{w} / \mathrm{v})$ chlorophyll standard.

3.2. Optimization of Time Needed to Remove Chlorophyll Maximally. Figure 2 showed the time dependence of the MIP in removing chlorophyll. Optimum chlorophyll removal as indicated by the leveling off of the plot was achieved at $6 \mathrm{~min}$ which is comparable to the fast kinetics exhibited by GCB (4.5 min) in removing chlorophyll [14].

3.2.1. Selectivity Experiments. To investigate selectivity, both the planar pesticides and the interfering species (chlorophyll) were exposed to the MIP as well as to GCB as single entities and in a mixture form at optimum conditions. In all cases the MIP did not bind any of the planar pesticides while the percentage of chlorophyll bound was over $99.75 \%$ (see Table 1). GCB on the other hand did not show any specific selectivity towards any of the analytes as it bound over $88 \%$ of each of the planar pesticides as well as the chlorophyll from their prepared standard solutions. It should be noted that the same experiments were performed with the NIP which also exhibited no specific selectivity as all of the analytes were bound just like with GCB. However the percentages bound by the NIP were very low, less than $3.7 \%$ in all cases, and as such were considered negligible.

GCB was able to bind higher quantities of the analytes than the NIP because it has a layered, planar structure which easily flushed with those of the planar pesticides. The NIPs on the other hand have a three-dimensional (3D) highly cross-linked structure with no recognition sites that could allow them to highly bind the pesticides or the chlorophyll. In addition to the imprints within the MIP structure, the 
high specificity of the prepared MIP to the chlorophyll was thought to be due to the bulkiness of the $3 \mathrm{D}$ structure of the chlorophyll locking with the $3 \mathrm{D}$, highly cross-linked MIP structure.

3.3. Effectiveness of Chlorophyll Removal from Spinach Leaves Extract. The initial chlorophyll absorbance of the methanolic, spinach extract was reduced from 0.683 to as low as $0.092 \mathrm{Au}$ after the addition of the MIP. For the traditional medicine extracts there was removal from a chlorophyll absorbance of 0.795 to $0.0897 \mathrm{Au}$ in Nymph lotus and 0.649 to $0.0899 \mathrm{Au}$ in Acalypha villicaulis. In all cases, the MIP had significantly removed the interfering chlorophyll to absorbance values far below what is regarded as the interfering chlorophyll which is the absorbance of a $2 \%$ $(\mathrm{w} / \mathrm{v})$ chlorophyll standard determined to be $0.401 \mathrm{Au}$ (the literature value is $0.399 \mathrm{Au}$ ) [1]. This was also marked by the loss of the green characteristic color of the plant extracts to almost colorless solutions after exposure to the MIP powder.

\section{Conclusion}

Based on these preliminary results it can be concluded that a promising, polymeric sorbent, that is, cheaper and with higher selectivity, would soon replace the challenged, expensive, and nonselective GCB in the dispersive SPE kits of QuEChERS. This would result in cheaper, improved, and reliable analytical results. A rough estimation of the cost of this MIP indicates that it would be at least half the price of the GCB (GCB is about \$ 50 per gram).

\section{Acknowledgments}

This work was supported by funding from Agilent Technologies (USA), Andrew Mellon Foundation, and STINT (Sweden (Lund University)-South Africa (Rhodes University) collaboration).

\section{References}

[1] http://www.sigmaaldrich.com/etc/medialib/docs/Supelco/ General_Information.

[2] S. J. Lehotay, K. Maštovská, and A. R. Lightfield, "Use of buffering and other means to improve results of problematic pesticides in a fast and easy method for residue analysis of fruits and vegetables," Journal of AOAC International, vol. 88, no. 2, pp. 615-629, 2005.

[3] J. Hajslova and J. Zrostlikova, "Matrix effects in (ultra)trace analysis of pesticide residues in food and biotic matrices," Journal of Chromatography A, vol. 1000, no. 1-2, pp. 181-197, 2003.

[4] M. Careri, L. Elviri, A. Mangia, and I. Zagnoni, "Rapid method for determination of chlormequat residues in tomato products by ion-exchange liquid chromatography/electrospray tandem mass spectrometry," Rapid Communications in Mass Spectrometry, vol. 16, no. 19, pp. 1821-1826, 2002.

[5] http://www.chem.agilent.com/Library/applications/59904248EN.pdf.
[6] C. Baggiani, L. Anfossi, and C. Giovannoli, "Molecular imprinted polymers as synthetic receptors for the analysis of myco- and phyco-toxins," Analyst, vol. 133, no. 6, pp. 719-730, 2008.

[7] C. He, Y. Long, J. Pan, K. Li, and F. Liu, "Application of molecularly imprinted polymers to solid-phase extraction of analytes from real samples," Journal of Biochemical and Biophysical Methods, vol. 70, pp. 133-150, 2007.

[8] V. Pichon, "Selective sample treatment using molecularly imprinted polymers," Journal of Chromatography A, vol. 1152, no. 1-2, pp. 41-53, 2007.

[9] F. G. Tamayo, E. Turiel, and A. Martín-Esteban, "Molecularly imprinted polymers for solid-phase extraction and solid-phase microextraction: recent developments and future trends," Journal of Chromatography A, vol. 1152, no. 1-2, pp. 32-40, 2007.

[10] V. Pichon and K. Haupt, "Affinity separations on molecularly imprinted polymers with special emphasis on solid-phase extraction," Journal of Liquid Chromatography \& Related Technologies, vol. 29, pp. 989-1023, 2006.

[11] E. Caro, R. M. Marcé, F. Borrull, P. A. G. Cormack, and D. C. Sherrington, "Application of molecularly imprinted polymers to solid-phase extraction of compounds from environmental and biological samples," Trends in Analytical Chemistry, vol. 25, no. 2, pp. 143-154, 2006.

[12] F. Qiao, H. Sun, H. Yan, and K. H. Row, "Molecularly imprinted polymers for solid phase extraction," Chromatographia, vol. 64, no. 11-12, pp. 625-634, 2006.

[13] B. S. Batlokwa, J. Mokgadi, T. Nyokong, and N. Torto, "Optimal template removal from molecularly imprinted polymers by pressurized hot water extraction," Chromatographia, vol. 73, no. 5-6, pp. 589-593, 2011.

[14] K. Mastovska and S. J. Lehotay, Pittcon Conference Proceedings, 2009. 

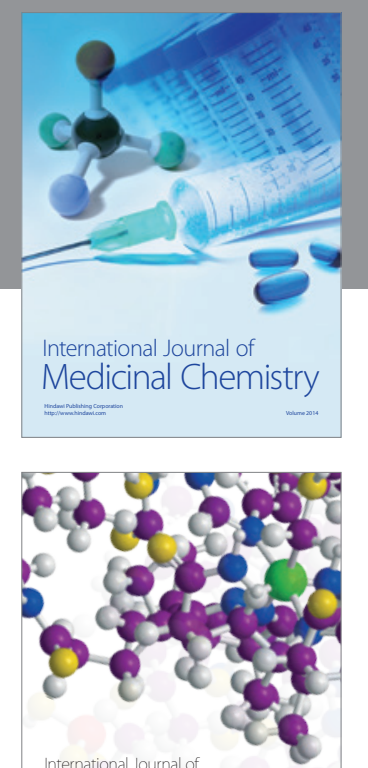

\section{Carbohydrate} Chemistry

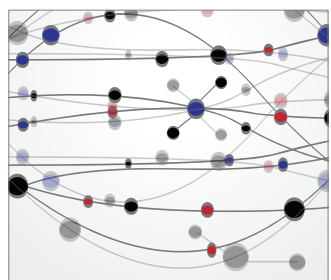

The Scientific World Journal
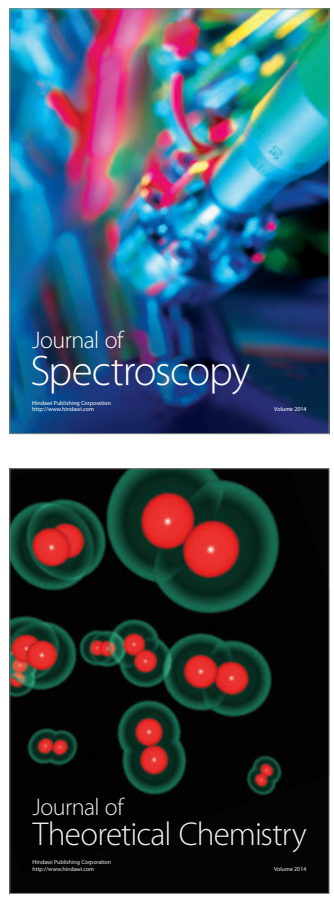
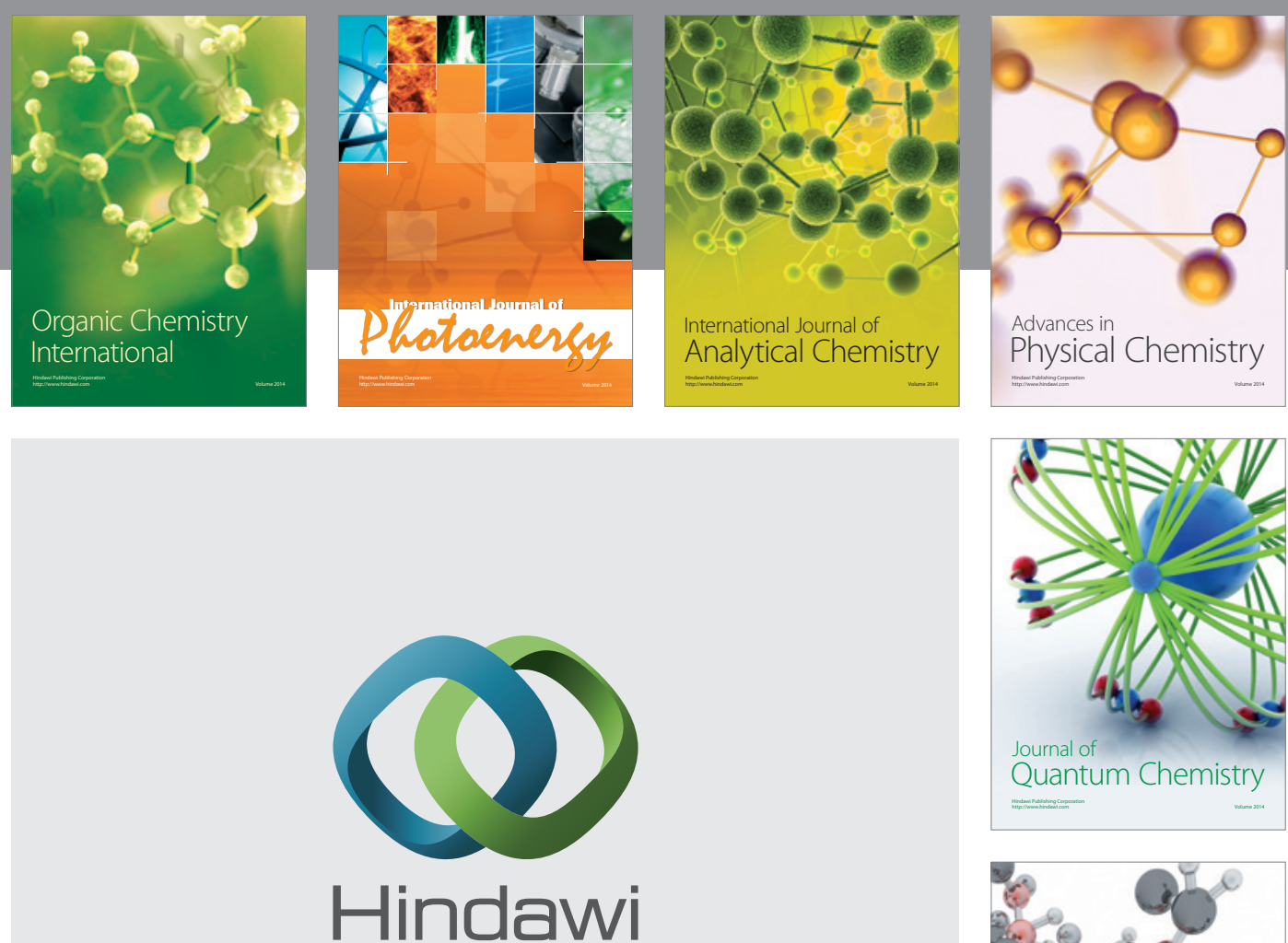

Submit your manuscripts at

http://www.hindawi.com

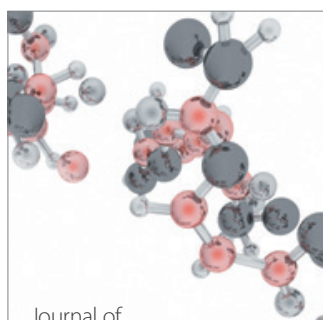

Analytical Methods

in Chemistry

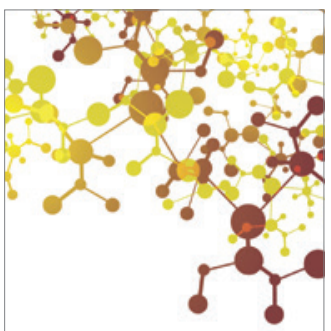

Journal of

Applied Chemistry

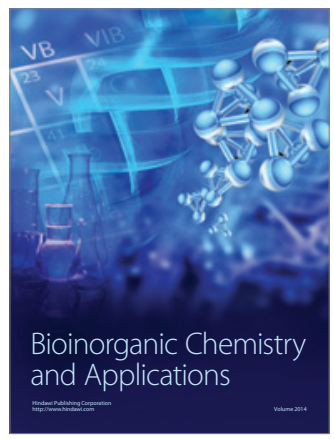

Inorganic Chemistry
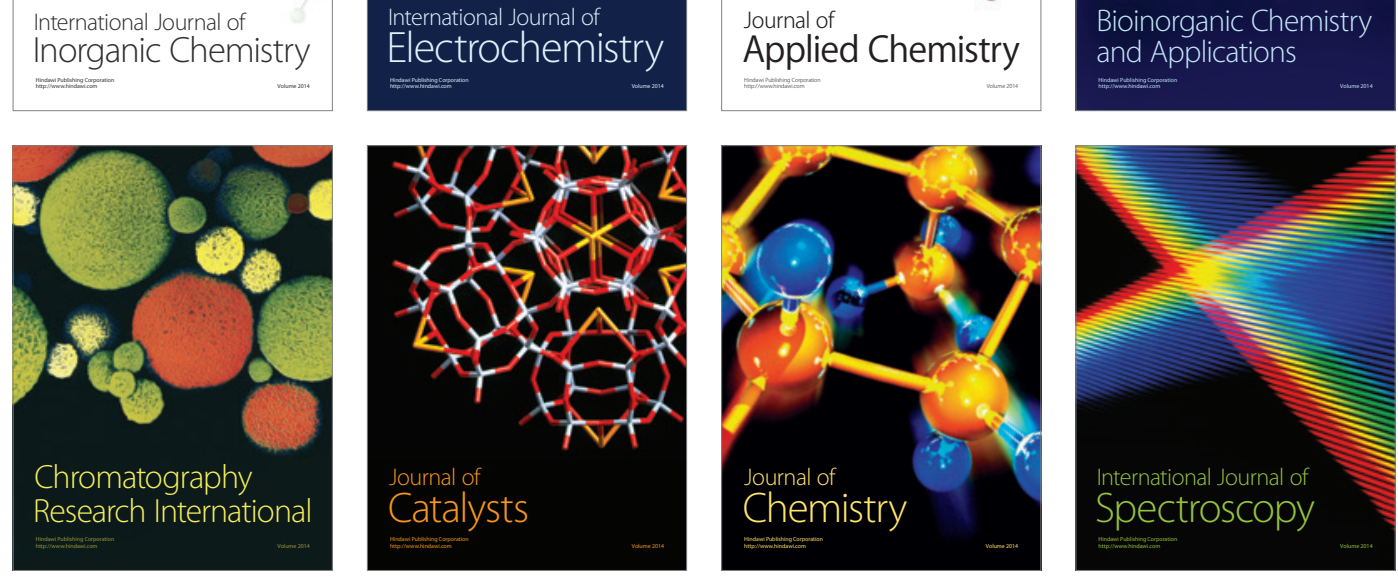